\title{
ON THE DISCRETE ASYMPTOTIC STABILITY CONDITIONS OF PERTURBED LINEAR DISCRETE SYSTEMS WITH PERIODIC COEFFICIENTS
}

\author{
KEMAL USLU ${ }^{1}$
}

(Received 8 June, 2005)

\begin{abstract}
We study the discrete asymptotic stability conditions of the perturbed system of first-order linear difference equations with periodic coefficients under the assumption that the related unperturbed system is discrete asymptotically stable. These conditions are dependent on the perturbation matrix $B(n)$ itself and a different parameter is given for obtaining some estimates for the solutions of the unperturbed system.
\end{abstract}

2000 Mathematics subject classification: primary 34D05, 34D10; secondary 34D20, $34 \mathrm{E} 10$.

Keywords and phrases: discrete asymptotic stability, linear difference systems with periodic coefficients, Lyapunov methods.

\section{Introduction}

In various fields of scientific endeavour such as physics, the study of neurology and electricity, we encounter linear first-order difference equations with periodic coefficients. Furthermore, such systems with periodic coefficients are adequate mathematical models for numerous processes and phenomena studied in robotics technology.

It is also an important issue to analyse perturbed systems of equations. For the perturbed systems of linear first-order discrete equations, the main goal is to find the conditions and regions of stability in terms of the perturbation matrix, and in particular, to find matrices that represent the transition from discrete stability to discrete instability.

The main purpose of this paper is to determine the discrete asymptotic stability conditions of the perturbed system of linear first-order difference equations with

\footnotetext{
'The University of Selcuk, Science and Art Faculty, Department of Mathematics, Campus/Konya, Turkey; e-mail: kuslu@selcuk.edu.tr.

(C) Australian Mathematical Society 2006, Serial-fee code 1446-1811/06
} 
periodic coefficients on the basis of some numeric characteristics obtained for an unperturbed system. The paper is organised as follows. In Section 2, we introduce some preliminary results and some numeric characteristics for the system of first-order linear discrete equations with periodic coefficients and compare these characteristics. In Section 3, we give a discrete asymptotic stability condition for the perturbed system by using methods from [3] and a different discrete stability parameter.

\section{Preliminaries}

Let us consider the system of linear difference equations with periodic coefficients

$$
x(n+1)=A(n) x(n), \quad n \geq 0,
$$

where $A(n)$ is a periodic $N \times N$ matrix with period $T$, that is, $A(n+T)=A(n)$, $n \geq 0$, for some positive integer $T$.

The zero solution of the system (2.1) is discrete asymptotically stable if and only if, from the spectral criterion, each eigenvalue of the monodromy matrix

$$
X(T)=A(T-1) A(T-2) \cdots A(1) A(0)=\prod_{j=0}^{T-1} A(j)
$$

of (2.1) has modulus less than 1 (that is, $\left|\lambda_{i}(X(T))\right|<1$ ) (see [5] for details).

However using the second method of Lyapunov, the computation of the eigenvalues is unnecessary. According to Lyapunov's second method, in particular, for the autonomous system (in the case of constant coefficients, $A(n)=A, n \geq 0$ ) the zero solution of (2.1) is discrete asymptotically stable if and only if for every positive Hermitian matrix $C_{1}$, the Lyapunov matrix equation

$$
A^{*} H A-H=-C_{1}, \quad C_{1}=C_{1}^{*}>0
$$

has a unique solution which is also Hermitian and positive definite. In this case a solution to (2.3) is defined by $H=\sum_{k=0}^{\infty}\left(A^{*}\right)^{k} C_{1} A^{k}$, where $A^{*}$ denotes the adjoint matrix of $A$ (see [5]). Indeed, if we allow $C_{1}$ to be the identity matrix $I$, then a solution of (2.3) is replaced by $H=\sum_{k=0}^{\infty}\left(A^{*}\right)^{k} A^{k}$. Thus, we can introduce the parameter

$$
w(A)=\|H\|
$$

which gives a characteristic of discrete asymptotic stability of the zero solution to the system $(2.1)$ for the autonomous case $[4,6]$. Therefore we can write the following inequality $[1,4,6]$ :

$$
\left\|A^{n}\right\| \leq\left(1-\frac{1}{w(A)}\right)^{n / 2} \sqrt{w(A)}, \quad n \geq 0 .
$$


It is noted that we henceforth consider the spectral norm of $N \times N$ square matrices $B$, that is, $\|B\|=\max _{\|x\|=1}\|B x\|$, where

$$
\|x\|=\left(\sum_{i=1}^{N}\left|x_{i}\right|^{2}\right)^{1 / 2}
$$

denotes the norm of a vector $x=\left(x_{1}, \ldots, x_{N}\right)$ in the linear Euclidian space $E_{N}$ with the inner product $\langle x, y\rangle=\sum_{i=1}^{N} x_{i} \bar{y}_{i}$, where the overbar denotes complex conjugation.

Now let us consider the initial value problem for (2.1)

$$
\begin{aligned}
x(n+1) & =A(n) x(n), \quad n \geq 0, \\
x(0) & =x_{0} .
\end{aligned}
$$

Assume that the matrix sequence $\{X(n)\}$ is a solution to the problem

$$
\begin{aligned}
X(n+1) & =A(n) X(n), \quad n \geq 0, \\
X(0) & =I .
\end{aligned}
$$

Then a solution $\{x(n)\}$ to the initial value problem (2.4) has the form of $x(n)=X(n) x_{0}$.

Similar to the constant coefficient case, consider the following matrix series given in [2]:

$$
F=\sum_{k=0}^{\infty}\left(X^{*}(T)\right)^{k} X(T)^{k} .
$$

Since the matrix $X(T)$ in (2.2) is the monodromy matrix; in view of the Lyapunov criterion, series (2.5) converges if and only if the zero solution to (2.1) is discrete asymptotically stable. We consider the first numeric characteristic for discrete asymptotic stability of the zero solutions to (2.1) to be the pair $\left\{w_{1}(A, T), \rho(A, T)\right\}$, where $w_{1}(A, T)=\|F\|$ and $\rho(A, T)=\max _{0 \leq m<T}\{\|X(m)\|\}$ (see [2] for details).

To give another characteristic for the discrete asymptotic stability of $(2.4)$, let us consider the matrix series given in [2]: $\Phi=\sum_{k=0}^{\infty} X^{*}(k) X(k)$. Then we can use the periodicity to rewrite $\Phi$ as

$$
\Phi=\sum_{k=0}^{\infty}\left[X^{*}(T)\right]^{k} C_{2}[X(T)]^{k}, \quad C_{2}=\sum_{s=0}^{T-1} X^{*}(s) X(s) .
$$

This matrix series (2.6) is a solution to the discrete Lyapunov equation

$$
X^{*}(T) \Phi X(T)-\Phi=-C_{2} .
$$

Since the matrix in (2.2) is the monodromy matrix, according to the Lyapunov criterion, series (2.6) converges if and only if the zero solution to (2.1) is discrete asymptotically 
stable. Thus the last numeric characteristic which we mentioned here for the discrete asymptotic stability of the zero solution to (2.1) appears to be the norm of the matrix series (2.6)

$$
w_{2}(A, T)=\|\Phi\|
$$

(see [2]).

A comparison of $w_{2}(A, T)$ with $w_{1}(A, T)$ and $\rho(A, T)$ obviously yields

$$
w_{1}(A, T) \leq w_{2}(A, T), \quad \rho(A, T) \leq \sqrt{w_{2}(A, T)}
$$

(see [2]).

\section{Main results}

Now we consider the linear perturbation system of difference equations with periodic coefficients

$$
y(n+1)=[A(n)+B(n)] y(n), \quad n \geq 0,
$$

where $A(n)$ and $B(n)$ are periodic matrices with period $T$.

Now our main goal is to obtain conditions on a perturbation matrix $B(n)$ for the discrete asymptotic stability of the zero solution to system (3.1) and prove the continuity of the norm of the matrix series (2.6). For this goal, we have obtained different results by using the methods given in [3].

Suppose that the following Cauchy problem:

$$
\begin{aligned}
Y(n+1) & =[A(n)+B(n)] Y(n), \quad n \geq 0, \\
Y(0) & =I
\end{aligned}
$$

has a solution of the matrix sequence $\{Y(n)\}$. Then a solution $\{y(n)\}$ with initial value $y(0)=y_{0}$ to system (3.1) is obviously in the form $y(n)=Y(n) y_{0}$. Therefore the monodromy matrix for (3.1) will be

$$
Y(T)=[A(T-1)+B(T-1)] \cdots[A(0)+B(0)]=\prod_{j=0}^{T-1}[A(j)+B(j)] .
$$

THEOREM 3.1. If the perturbation matrix $B(n)$ is such that

$$
\|Y(T)-X(T)\|=\sqrt{\|X(T)\|^{2}+\frac{\sum_{s=0}^{T-1}\|X(s)\|^{2}}{w_{2}(A, T)}}-\|X(T)\|,
$$

then the zero solution to system (3.1) is discrete asymptotically stable. 
PROOF. The inequality (3.2) is equivalent to

$$
\begin{aligned}
\beta= & \sum_{s=0}^{T-1}\|X(s)\|^{2} \\
& -\left(-2\|X(T)\|\|Y(T)-X(T)\|+\|Y(T)-X(T)\|^{2}\right)\|\Phi\|>0 .
\end{aligned}
$$

By using periodicity, we write $Y(k T)=[Y(T)]^{k}$ for $k \geq 0$. Thus the sequence $\{Y(k T)\}$ is the solution to the problem

$$
\begin{aligned}
Y((k+1) T) & =[X(T)+Y(T)-X(T)] Y(k T), \quad k \geq 0, \\
Y(0) & =I .
\end{aligned}
$$

For the vector $v$ of $E_{N}$, consider the form $l=\langle\Phi Y((k+1) T) v, Y((k+1) T) v\rangle$.

From (2.6) and (3.4) we obtain

$$
\begin{aligned}
l= & \langle\Phi Y(k T) v, Y(k T) v\rangle-\left\langle\left(\sum_{s=0}^{T-1} X^{*}(s) X(s)\right) Y(k T) v, Y(k T) v\right\rangle \\
& +\left\langle\left(\begin{array}{c}
X^{*}(T) \Phi(Y(T)-X(T)) \\
+(Y(T)-X(T))^{*} \Phi X(T) \\
+(Y(T)-X(T))^{*} \Phi(Y(T)-X(T))
\end{array}\right) Y(k T) v, Y(k T) v\right\rangle .
\end{aligned}
$$

Using (3.3), we have $l \leq(1-\beta /\|\Phi\|)\langle\Phi Y(k T) v, Y(k T) v\rangle$. Finally, we get

$$
\langle\Phi Y(k T) v, Y(k T) v\rangle \leq\left(1-\frac{\beta}{\|\Phi\|}\right)^{k}\langle\Phi v, v\rangle .
$$

Since the matrix $\Phi$ is positive definite, we obtain

$$
\left\|(Y(T))^{k} v\right\|=\|Y(k T) v\| \rightarrow 0, \quad k \rightarrow \infty,
$$

for every vector $v \in E_{N}$. Then the spectrum of the monodromy matrix $Y(T)$ must lie in the unit disk $\left\{\left|\lambda_{i}(Y(T))\right|<1, i=1, \ldots, N\right\}$. This implies that the zero solution of system (3.1) is discrete asymptotically stable.

COROLLARY 3.2. Let

$$
a=\max _{0 \leq j<T}\{\|A(j)\|\} \text { and } b=\max _{0 \leq j<T}\{\|B(j)\|\} .
$$

If the perturbation matrix $B(n)$ is such that

$$
T b(a+b)^{T-1}<\sqrt{\|X(T)\|^{2}+\frac{\sum_{s=0}^{T-1}\|X(s)\|^{2}}{w_{2}(A, T)}}-\|X(T)\|,
$$

then the zero solution to system (3.1) is discrete asymptotically stable. 
Proof. It is easy to show that

$$
\|Y(T)-X(T)\| \leq(a+b)^{r}-a^{T} \leq T b(a+b)^{T-1} .
$$

Therefore, the proof for discrete asymptotic stability of the zero solution to system (3.1) is immediate from (3.2) and (3.6).

Suppose that for the perturbation matrix $B(n)$, the condition in Theorem 3.1 is satisfied. Then the zero solution to system (3.1) is discrete asymptotically stable. Thus we can consider the matrix series

$$
\hat{\Phi}=\sum_{k=0}^{\infty}\left[Y^{*}(T)\right]^{k} C_{2}[Y(T)]^{k} .
$$

Since the matrix $\hat{\Phi}$ is a solution to the discrete Lyapunov matrix equation

$$
Y^{*}(T) \hat{\Phi} Y(T)-\hat{\Phi}=-C_{2},
$$

by analogy with (2.8), we define the numeric characteristic for asymptotic stability of the zero solution (3.1) to be $w_{2}(A+B, T)=\|\hat{\Phi}\|$.

THEOREM 3.3. If $\Phi$ and $\hat{\Phi}$ are the solutions of Lyapunov matrix equations (2.7) and (3.8) respectively, then

$$
\frac{\|\Phi-\hat{\Phi}\|}{\|\Phi\|} \leq \frac{\left(\sum_{s=0}^{T-1}\|X(s)\|^{2}\right)-\beta}{1-\left(\left(\sum_{s=0}^{T-1}\|X(s)\|^{2}\right)-\beta\right)},
$$

where the constant $\beta$ is defined by (3.3).

Proof. Using (2.7) and (3.8), we write $Y^{*}(T)(\hat{\Phi}-\Phi) Y(T)-(\hat{\Phi}-\Phi)=-C_{2}$, where

$$
\begin{aligned}
C_{2}= & X^{*}(T) \Phi(Y(T)-X(T))+(Y(T)-X(T))^{*} \Phi X(T) \\
& +(Y(T)-X(T))^{*} \Phi(Y(T)-X(T)) .
\end{aligned}
$$

The discrete Lyapunov matrix equation (2.7) has a unique solution

$$
\hat{\Phi}-\Phi=\sum_{k=0}^{\infty}\left[Y^{*}(T)\right]^{k} C_{2}[Y(T)]^{k} .
$$

So, from (3.7), we get

$$
\|\hat{\Phi}-\Phi\| \leq\left\|C_{2}\right\|\|\hat{\Phi}\| \leq\left\|C_{2}\right\|\|\hat{\Phi}-\Phi\|+\left\|C_{2}\right\|\|\Phi\| .
$$


Clearly,

$$
\begin{aligned}
\left\|C_{2}\right\| & \leq\left(2\|X(T)\|\|Y(T)-X(T)\|+\|Y(T)-X(T)\|^{2}\right)\|\Phi\| \\
& =\left(\sum_{s=0}^{T-1}\|X(s)\|^{2}\right)-\beta,
\end{aligned}
$$

thus this completes the statement of (3.9).

COROLLARY 3.4. If the perturbation matrix is such that

$$
\|Y(T)-X(T)\| \leq \sqrt{\|X(T)\|^{2}+\frac{\sum_{s=0}^{T-1}\|X(s)\|^{2}}{4 w_{2}(A, T)}}-\|X(T)\|
$$

holds with the condition

$$
\sum_{s=0}^{T-1}\|X(s)\|^{2} \leq 2
$$

then $\|\Phi-\hat{\Phi}\| /\|\Phi\|<1$.

Proof. From (3.10) and (3.11), we obtain

$$
\begin{aligned}
\frac{\|\Phi-\hat{\Phi}\|}{\|\Phi\|} & \leq \frac{\left(2\|X(T)\|\|Y(T)-X(T)\|+\|Y(T)-X(T)\|^{2}\right)\|\Phi\|}{1-\left(2\|X(T)\|\|Y(T)-X(T)\|+\|Y(T)-X(T)\|^{2}\right)\|\Phi\|} \\
& <\frac{\frac{1}{4 w_{2}(A, T)} \sum_{s=0}^{T-1}\|X(s)\|^{2}}{1-\frac{1}{4 w_{2}(A, T)}\left(\sum_{s=0}^{T-1}\|X(s)\|^{2}\right) w_{2}(A, T)} \\
& =\frac{\frac{1}{4} \sum_{s=0}^{T-1}\|X(s)\|^{2}}{1-\frac{1}{4} \sum_{s=0}^{T-1}\|X(s)\|^{2}}<1 .
\end{aligned}
$$

Using (3.12) in the last inequality, the statement of the corollary follows.

\section{References}

[1] O. Akın and H. Bulgak, "Linear difference equations and stability theory", (Selcuk Univ. Research Centre of Applied Mathematics, Konya, 1998).

[2] K. Aydın, H. Bulgak and G. V. Demidenko, "Numeric characteristics for asymptotic stability of solutions to linear difference equations with periodic coefficients", Siberian Math. J. 41 (2000) 1005-1014. 
[3] K. Aydin, H. Bulgak and G. V. Demidenko, "Continuity of numeric characteristics for asymptotic stability of solutions to linear difference equations with periodic coefficients", Selcuk J. Appl. Math. 2 (2001) 5-10.

[4] A. Ya. Bulgakov and S. K. Godunov, "Circular dichotomy of the matrix spectrum", Sibirsk. Mat. Zh. 29 (1988) 59-70.

[5] S. N. Elaydi, An Introduction to Difference Equations (Springer, New York, 1996).

[6] S. K. Godunov, Modern Aspects of Linear Algebra, Translations of Mathematical Monographs 175 (American Mathematical Society, Providence, RI, 1998). 